\title{
Statistics of the number of equilibria in random social dilemma evolutionary games with mutation
}

\author{
Manh Hong Duong ${ }^{1, \mathrm{a}}$ (D) and The Anh $\operatorname{Han}^{2, \mathrm{~b}}$ \\ 1 School of Mathematics, University of Birmingham, Birmingham B15 2TT, UK \\ 2 School of Computing, Engineering and Digital Technologies, Teesside University, Middlesbrough TS1 3BX, UK
}

Received 14 July 2021 / Accepted 9 August 2021 / Published online 23 August 2021

(C) The Author(s) 2021

\begin{abstract}
In this paper, we study analytically the statistics of the number of equilibria in pairwise social dilemma evolutionary games with mutation where a game's payoff entries are random variables. Using the replicator-mutator equations, we provide explicit formulas for the probability distributions of the number of equilibria as well as other statistical quantities. This analysis is highly relevant assuming that one might know the nature of a social dilemma game at hand (e.g., cooperation vs coordination vs anticoordination), but measuring the exact values of its payoff entries is difficult. Our delicate analysis shows clearly the influence of the mutation probability on these probability distributions, providing insights into how varying this important factor impacts the overall behavioural or biological diversity of the underlying evolutionary systems.
\end{abstract}

\section{Introduction}

\subsection{The replicator-mutator equation}

The replicator-mutator equation is a set of differential equations describing the evolution of frequencies of different strategies in a population that takes into account both selection and mutation mechanisms. It is a powerful and important tool given that in many social and, more generally, biological settings mutation is non-negligible $[1,23,30,37,38,40]$. It has been employed in the study of, among other applications, population genetics [12], autocatalytic reaction networks [34], language evolution [25], the evolution of cooperation $[17,24]$ and dynamics of behavior in social networks [26].

Suppose that in an infinite population there are $n$ types/strategies $S_{1}, \ldots, S_{n}$ whose frequencies are, respectively, $x_{1}, \ldots, x_{n}$. These types undergo selection; that is, the reproduction rate of each type, $S_{i}$, is determined by its fitness or average payoff, $f_{i}$, which is obtained from interacting with other individuals in the population. The interaction of the individuals in the population is carried out within randomly selected groups of $d$ participants (for some integer $d$ ). That is, they play and obtain their payoffs from a $d$-player game, defined by a payoff matrix. We consider here symmetric games where the payoffs do not depend on the ordering of the players in a group. Mutation is included by adding the possibility that individuals spontaneously

\footnotetext{
${ }^{a}$ e-mail: h.duong@bham.ac.uk (corresponding author)
}

b e-mail: T.Han@tees.ac.uk change from one strategy to another, which is modeled via a mutation matrix, $Q=\left(q_{j i}\right), j, i \in\{1, \ldots, n\}$. The entry $q_{j i}$ denotes the probability that a player of type $S_{j}$ changes its type or strategy to $S_{i}$. The mutation matrix $Q$ is a row-stochastic matrix, i.e.,

$$
\sum_{j=1}^{n} q_{j i}=1, \quad 1 \leq i \leq n .
$$

The replicator-mutator is then given by, see e.g. [18$20,28]$

$$
\begin{aligned}
& \dot{x}_{i}=\sum_{j=1}^{n} x_{j} f_{j}(\mathbf{x}) q_{j i}-x_{i} \bar{f}(\mathbf{x})=: g_{i}(x), \\
& i=1, \ldots, n
\end{aligned}
$$

where $\mathbf{x}=\left(x_{1}, x_{2}, \ldots, x_{n}\right)$ and $\bar{f}(\mathbf{x})=\sum_{i=1}^{n} x_{i} f_{i}(\mathbf{x})$ denotes the average fitness of the whole population. The replicator dynamics is a special instance of (1) when the mutation matrix is the identity matrix.

\subsection{The replicator-mutator equation for two-player two-strategy games}

In particular, for two-player two-strategy games with a payoff matrix

$$
\begin{array}{ccc} 
& S_{1} & S_{2} \\
S_{1} & \left(\begin{array}{ll}
a_{11} & a_{12} \\
a_{21} & a_{22}
\end{array}\right)
\end{array}
$$


where $a_{i j}, i, j \in\{1,2\}$ is the payoff that a player using strategy $S_{i}$ obtains when interacting with another player using strategy $S_{j}$, the replicator-mutator equation is given by

$$
\begin{aligned}
\dot{x}= & q_{11} a_{11} x^{2}+q_{11} x(1-x) a_{12} \\
& +q_{21} x(1-x) a_{21}+q_{21} a_{22}(1-x)^{2} \\
& -x\left(a_{11} x^{2}+\left(a_{12}\right.\right. \\
& \left.\left.+a_{21}\right) x(1-x)+a_{22}(1-x)^{2}\right) .
\end{aligned}
$$

Here $x$ is the frequency of the first strategy and $1-x$ is the frequency of the second one. Using the identities $q_{11}=q_{22}=1-q, \quad q_{12}=q_{21}=q$, Eq. (2) becomes

$$
\begin{aligned}
\dot{x}= & \left(a_{12}+a_{21}-a_{11}-a_{22}\right) x^{3} \\
& +\left(a_{11}-a_{21}-2\left(a_{12}-a_{22}\right)\right. \\
& \left.+q\left(a_{22}+a_{12}-a_{11}-a_{21}\right)\right) x^{2} \\
& +\left(a_{12}-a_{22}+q\left(a_{21}-a_{12}-2 a_{22}\right)\right) x \\
& +q a_{22} .
\end{aligned}
$$

Two-player social dilemma games. In this paper, we focus on two-player (i.e. pairwise) social dilemma games. They have provided a main framework to study several important issues such as the evolution of cooperative behavior and its supporting mechanisms $[13,24,29,31]$. We adopt the following parameterized payoff matrix to study the full space of two-player social dilemma games where the first strategy is cooperator and second is defector [31,36,39], $a_{11}=1 ; a_{22}=0$; $0 \leq a_{21}=T \leq 2$ and $-1 \leq a_{12}=S \leq 1$, that covers the following games

(i) the Prisoner's Dilemma (PD): $2 \geq T>1>0>$ $S \geq-1$

(ii) the Snow-Drift (SD) game: $2 \geq T>1>S>0$,

(iii) the Stag Hunt (SH) game: $1>T>0>S \geq-1$,

(iv) the Harmony (H) game: $1>T \geq 0,1 \geq S>0$.

In this paper, we are interested in random social dilemma games where $T$ and $S$ are uniform random variables in the corresponding intervals, namely

- In PD games: $T \sim U(1,2), S \sim U(-1,0)$,

- In SD games: $T \sim U(1,2), S \sim U(0,1)$,

- In SH games: $T \sim U(0,1), S \sim U(-1,0)$,

- In H games: $T \sim U(0,1), S \sim U(0,1)$.

Random evolutionary games, in which the payoff entries are random variables, have been employed extensively to model social and biological systems in which very limited information is available, or where the environment changes so rapidly and frequently that one cannot describe the payoffs of their inhabitants' interactions
[4-7, 9, 11, 15, 22]. Equilibrium points of such evolutionary system are the compositions of strategy frequencies where all the strategies have the same average fitness. Biologically, they predict the co-existence of different types in a population and the maintenance of polymorphism.

In this paper, we are interested in computing the probability distributions of the number of equilibria, which is a random variable, in the above random social dilemmas. Answering this question is of great importance in the context of social dilemmas since one might know the nature of the game, i.e. the payoff entries ranking in the game, but it might be difficult to predict or measure the exact values of these entries. When the mutation is absent $(q=0)$, the answer is trivial because there is always a fixed number of equilibria depending on the nature of the social dilemmas [8,24,32]. As shown in our analysis below, this is however not the case any longer when mutation is non-negligible, and this number highly depends on the nature of the social dilemma too.

The following result obtained in [8] provides explicit formulas for the probabilities $p_{i}^{G}$ that a game $G \in$ $\{\mathrm{SD}, \mathrm{H}, \mathrm{SH}, \mathrm{PD}\}$ has $i \in\{1,2,3\}$ equilibria.

Theorem 1 Suppose that $S$ and $T$ are uniformly distributed in the corresponding intervals as above. Then

$$
\begin{aligned}
& -p_{1}^{\mathrm{SD}}=p_{3}^{\mathrm{SD}}=0, \quad p_{2}^{\mathrm{SD}}=1 . \\
& -p_{1}^{\mathrm{H}}=p_{3}^{\mathrm{H}}=0, \quad p_{2}^{\mathrm{H}}=1 . \\
& -p_{2}^{\mathrm{SH}}=\frac{q}{2(1-q)} . \\
& -p_{2}^{\mathrm{PD}}=\left\{\begin{array}{l}
\frac{3 q}{2(1-q)} \quad \text { if } \quad 0<q \leq 1 / 3, \\
3-\frac{1}{2 q(1-q)} \quad \text { if } 1 / 3 \leq q \leq 1 / 2 .
\end{array}\right.
\end{aligned}
$$

According to the above theorem, SD games and $\mathrm{H}$ games are simple and complete. However, the probabilities of having 3 equilibria (or alternatively 1 equilibrium) in SH and $\mathrm{PD}$ games are left open in [8]. The key challenge is that the conditions for these games to have 3 equilibria (or alternatively 1 equilibrium) are much more complicated than those of 2 equilibria. The aim of this paper is to complete the above theorem, providing explicit formulas for these probabilities. As such, it will also allow us to derive other statistical quantities (such as average and variance), which are important to understand the overall distribution and complexity of equilibrium points in pairwise social dilemmas (with mutation). To this goal, we employ suitable changes of variables, which transform the problem of computing the probabilities to calculating areas, and perform delicate analysis.

\subsection{Main results}

The main result of this paper is the following.

Theorem 2 The probability that $S H$ and $P D$ games have 3 equilibria is given by, respectively 


$$
\begin{aligned}
& p_{3}^{\mathrm{SH}}=\left\{\begin{aligned}
1- & \frac{q}{2(1-q)}-\frac{1}{1-2 q}\left[\frac{(3 \sqrt{q}+2)^{2} \sqrt{q}\left(5 q^{3 / 2}+3 q^{2}-9 q-3 \sqrt{q}+4\right)}{12(\sqrt{q}+1)^{3}}\right. \\
& \left.\quad+\frac{-27 q^{3}-18 q^{2}-32 \sqrt{1-2 q} q+48 q+16 \sqrt{1-2 q}-16}{12 q}\right], \quad 0<q \leq 4 / 9, \\
1- & \frac{q}{2(1-q)}-\frac{8 \sqrt{q}(1-2 q)^{2}}{3(1-q)^{3}}, \quad 4 / 9<q<0.5
\end{aligned}\right. \\
& p_{3}^{\mathrm{PD}}=\left\{\begin{array}{l}
\frac{1}{1-2 q}\left[-\frac{2\left(q^{3}+3 q^{2}+(4 \sqrt{1-2 q}-6) q-2 \sqrt{1-2 q}+2\right)}{3 q}-\frac{1}{2} \frac{q^{3}}{(1-q)}\right], \quad 0 \leq q \leq \frac{3-\sqrt{5}}{2}, \\
\frac{-16(\sqrt{1-2 q}-1) q^{3 / 2}+2 q^{5 / 2}+15 q^{3}+(8 \sqrt{1-2 q}-25) q^{2}+q-8 \sqrt{1-2 q}+2 \sqrt{q}(8 \sqrt{1-2 q}-5)+5}{6(\sqrt{q}-1)^{3}\left(q^{3 / 2}+q\right)}, \frac{3-\sqrt{5}}{2}<q \leq 4 / 9, \\
\frac{1}{2} \frac{(1-2 q)^{2}}{q(1-q)}, \quad 4 / 9<q<0.5 .
\end{array}\right.
\end{aligned}
$$

The above theorem combines Theorem 3 (for SHgames) and Theorem 4 (for PD games), see Sect. 2. Theorems 1 and 2 provides explicitly the probability distributions of the number of equilibria for all the abovementioned pairwise social dilemmas. In SH-games and PD-games, these distributions are much more complicated and significantly depend on the mutation strength. We summarize these results in the following summary box.
As a consequence, we can now derive other statistical quantities such as the mean value, ENoE, and the variance, VarNoE of the number of equilibria using the following formulas

\section{Box 1: Probability of having $k$ equilibria in a pairwise social dilemma $\left(p_{k}\right)$}

- Snow Drift (SD)

$$
p_{1}=0, \quad p_{2}=1, \quad p_{3}=0 .
$$

\section{- Harmony game (H)}

$$
p_{1}=0, \quad p_{2}=1, \quad p_{3}=0 .
$$

\section{- Stag-Hunt game (SH)}

$$
\begin{aligned}
& p_{1}=1-p_{2}-p_{3} . \\
& p_{2}=\frac{q}{2(1-q)} \\
& p_{3}=\left\{\begin{array}{rr}
1-\frac{q}{2(1-q)}-\frac{1}{1-2 q}\left[\frac{(3 \sqrt{q}+2)^{2} \sqrt{q}\left(5 q^{3 / 2}+3 q^{2}-9 q-3 \sqrt{q}+4\right)}{12(\sqrt{q}+1)^{3}},\right. \\
\left.\quad+\frac{-27 q^{3}-18 q^{2}-32 \sqrt{1-2 q} q+48 q+16 \sqrt{1-2 q}-16}{12 q}\right], 0<q \leq 4 / 9 \\
1-\frac{q}{2(1-q)}-\frac{8 \sqrt{q}(1-2 q)^{2}}{3(1-q)^{3}}, & 4 / 9<q<0.5 .
\end{array}\right.
\end{aligned}
$$

\section{- Prisoner's Dilemma (PD)}

$$
\begin{aligned}
& p_{1}=1-p_{2}-p_{3} . \\
& p_{2}= \begin{cases}\frac{3 q}{2(1-q)} \quad \text { if } \quad 0<q \leq 1 / 3 \\
3-\frac{1}{2 q(1-q)} \quad \text { if } \quad 1 / 3 \leq q \leq 1 / 2 .\end{cases} \\
& p_{3}= \begin{cases}\frac{1}{1-2 q}\left[-\frac{2\left(q^{3}+3 q^{2}+(4 \sqrt{1-2 q}-6) q-2 \sqrt{1-2 q}+2\right)}{3 q}-\frac{1}{2} \frac{q^{3}}{(1-q)}\right], 0 \leq q \leq \frac{3-\sqrt{5}}{2}, \\
\frac{-16(\sqrt{1-2 q}-1) q^{3 / 2}+2 q^{5 / 2}+15 q^{3}+(8 \sqrt{1-2 q}-25) q^{2}+q-8 \sqrt{1-2 q}+2 \sqrt{q}(8 \sqrt{1-2 q}-5)+5}{6(\sqrt{q}-1)^{3}\left(q^{3 / 2}+q\right)}, \frac{3-\sqrt{5}}{2}<q \leq 4 / 9, \\
\frac{1}{2} \frac{(1-2 q)^{2}}{q(1-q)}, \quad 4 / 9<q<0.5 .\end{cases}
\end{aligned}
$$


Probability of having $k$ equilibria $\left(p_{k}\right)$ in a social dilemma
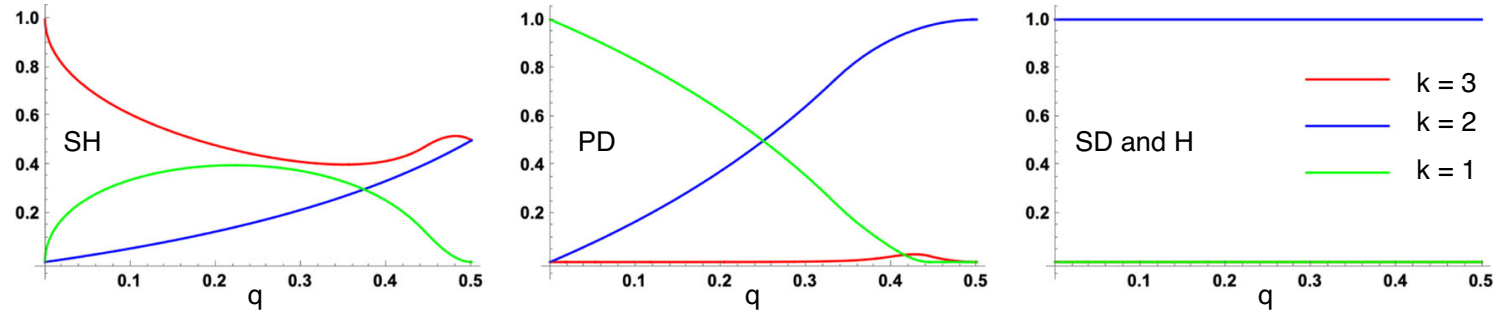
Average and variance of the number of
equilibria in a social dilemma

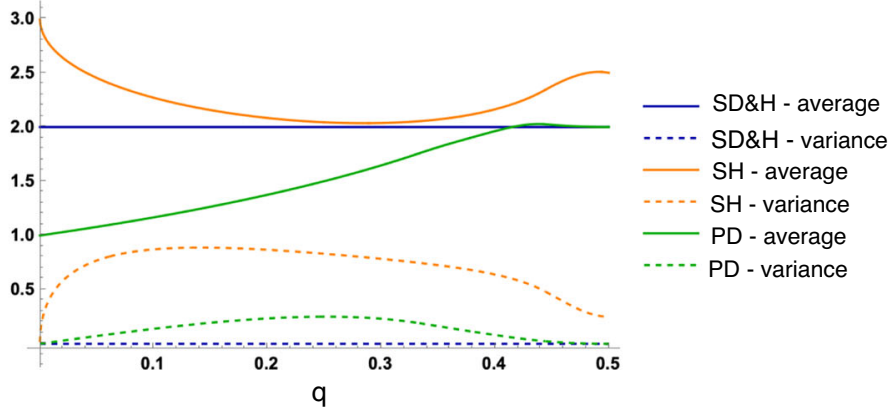

Fig. 1 Non-trivial effect of non-negligible mutations on the number of equilibria in a pairwise social dilemma. We show its statistics as a function of the mutation probability $q$ : probability of having $k(k=1,2,3)$ equilibria (top row), average and variance (bottom row). We look at all the four pairwise games, from left to right: SH, PD, SH and $\mathrm{H}$. The probability of having the maximal possible number of equilibria, i.e. $p_{3}$, is highest for $\mathrm{SH}$. It is very small for PD and always equals 0 for $\mathrm{SD}$ and $\mathrm{H}$ games. The probability of having two equilibria, $p_{2}$, is highest for $\mathrm{SD}$ and $\mathrm{H}$. As a result, $\mathrm{SH}$ has the highest average number of equilibria across all games, for all $0<q<0.5$ (it is minimum when $q=0.285$ ). For most $q$, PD has the lowest number of equilibria (it is maximum when $q=0.438$ ). All the figures are generated using analytical formulas derived in Box 1 (for $p_{k}$ ) and Eq. (4) for the average and variance. These analytical results are also in accordance with numerical simulation results provided in [8], obtained through samplings of the random payoff entries $T$ and $S$

$$
\begin{aligned}
\mathrm{ENoE} & =\sum_{i=1}^{3} i p_{i}, \\
\operatorname{VarNoE} & =\sum_{i=1}^{3} p_{i}(i-\mathrm{ENoE})^{2} .
\end{aligned}
$$

We depict these quantities in Fig. 1 below. Our delicate analysis clearly shows the influence of the mutation on the probability distributions, thus on the complexity and bio-diversity of the underlying evolutionary systems. We believe that our analysis may be used as exemplary material for teaching foundational courses in evolutionary game theory, computational/quantitative biology and applied probability.

The rest of the paper is organized as follows. In Sect. 2 , after recalling some preliminary details, we present the proof of the main theorem 2, which we split into Theorem 3 for $\mathrm{SH}$ games in Sect. 2.3 and Theorem 4 for PD games in Sect. 2.4. Finally, in Sect. 3 we provide further discussion.

\section{Probability of having three equilibria in $\mathrm{SH}$ and PD games}

\subsection{Joint probability density via change of variable}

The following lemma is a well-known result to compute the probability density of random variables using change of variables. We state here for two random variables that are directly applicable to our analysis, but the result is true in higher dimensional spaces.

Lemma 1 (Joint probability density via change of variable, [10, Section 3.3]) Suppose $\left(X_{1}, X_{2}\right)$ has joint density $f\left(x_{1}, x_{2}\right)$. Let $\left(Y_{1}, Y_{2}\right)$ be defined by $Y_{1}=$ $u_{1}\left(X_{1}, X_{2}\right)$ and $Y_{2}=u_{2}\left(X_{1}, X_{2}\right)$. Suppose that the map $\left(X_{1}, X_{2}\right) \rightarrow\left(Y_{1}, Y_{2}\right)$ is invertible with $X_{1}=v_{1}\left(Y_{1}, Y_{2}\right)$ and $X_{2}=v_{2}\left(Y_{1}, Y_{2}\right)$. Then the joint probability distribution of $Y_{1}$ and $Y_{2}$ is

$$
g\left(y_{1}, y_{2}\right)=|J| f\left(v_{1}\left(y_{1}, y_{2}\right), v_{2}\left(y_{1}, y_{2}\right)\right)
$$


where the Jacobian $J$ is given by

$$
J=\left|\begin{array}{ll}
\frac{\partial v_{1}\left(y_{1}, y_{2}\right)}{\partial y_{1}} & \frac{\partial v_{1}\left(y_{1}, y_{2}\right)}{\partial y_{2}} \\
\frac{\partial v_{2}\left(y_{1}, y_{2}\right)}{\partial y_{1}} & \frac{\partial v_{2}\left(y_{1}, y_{2}\right)}{\partial y_{2}}
\end{array}\right| .
$$

\subsection{Equilibria in social dilemmas}

By simplifying the right hand side of (3), equilibria of a social dilemma game are roots in the interval $[0,1]$ of the following cubic equation

$$
\begin{aligned}
& (T+S-1) x^{3}+(1-T-2 S+q(S-1-T)) x^{2} \\
& \quad+(S+q(T-S)) x=0 .
\end{aligned}
$$

It follows that $x=0$ is always an equilibrium. If $q=0$, (5) reduces to

$$
(T+S-1) x^{3}+(1-T-2 S) x^{2}+S x=0,
$$

which has solutions

$$
x=0, \quad x=1, \quad x^{*}=\frac{S}{S+T-1} .
$$

Note that for SH-games and SH-games $x^{*} \in(0,1)$, thus it is always an equilibrium. On the other hand, for PDgames and $\mathrm{H}$-games, $x^{*} \notin(0,1)$, thus it is not an equilibrium

If $q=\frac{1}{2}$ then the above equation has two solutions $x_{1}=\frac{1}{2}$ and $x_{2}=\frac{T+S}{T+S-1}$. In $\mathrm{PD}, \mathrm{SD}$ and $\mathrm{H}$ games, $x_{2} \notin(0,1)$, thus they have two equilibria $x_{0}=0$ and $x_{1}=\frac{1}{2}$. In the SH game: if $T+S<0$ then the game has three equilibria $x_{0}=0, x_{1}=\frac{1}{2}$ and $0<x_{2}<1$; if $T+S \geq 0$ then the game has only two equilibria $x_{0}=0, x_{1}=\frac{1}{2}$.

Now we consider the case $0<q<\frac{1}{2}$. For nonzero equilibrium points we solve the following quadratic equation

$$
\begin{aligned}
h(x):= & (T+S-1) x^{2}+(1-T-2 S+q(S-1-T)) x \\
& +S+q(T-S) \\
= & : a x^{2}+b x+c=0 .
\end{aligned}
$$

Set $t:=\frac{x}{1-x}$, then we have

$$
\begin{aligned}
\frac{h(x)}{(1-x)^{2}} & =(a+b+c) t^{2}+(b+2 c) t+c \\
& =-q t^{2}+(-q-a+c) t+c \\
& :=g(t)
\end{aligned}
$$

Therefore, a social dilemma has three equilibria iff $h$ has two distinct roots in $(0,1)$, which is equivalent to $g$ having two distinct positive roots, or the following conditions must hold

$$
\Delta=(q+a-c)^{2}+4 q c>0,
$$

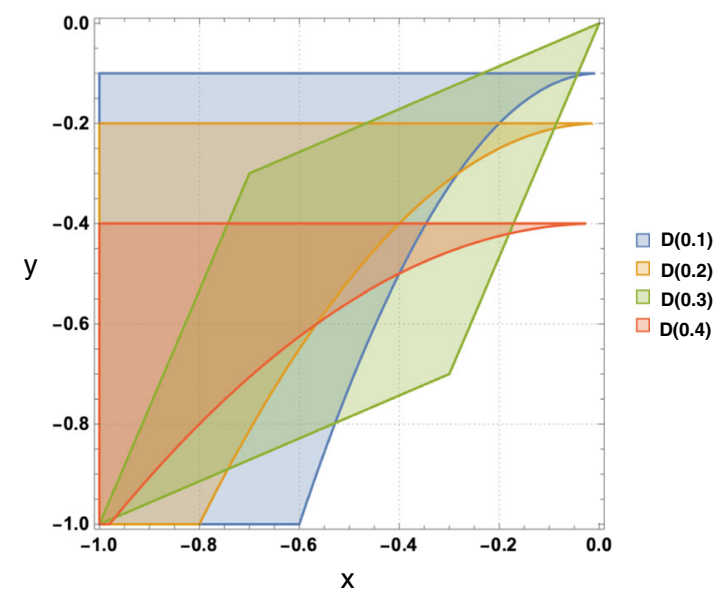

Fig. 2 Region $D$ are shown for different values of $q$, namely, $q=0.1,0.2,0.3$ and 0.4 , for illustration (see Sect. $2.2)$

$$
q+a-c<0, \quad c<0
$$

Let $\hat{T}:=T-1$ and

$$
\begin{aligned}
X(\hat{T}, S) & :=q+a-c=(1-q)(T-1)+q S \\
& =(1-q) \hat{T}+q S, \\
Y(\hat{T}, S) & :=c-q=q(T-1)+(1-q) \\
& =q \hat{T}+(1-q) S .
\end{aligned}
$$

The inverse transformation $(X, Y) \rightarrow(\hat{T}, S)$ is given by

$$
\begin{aligned}
& \hat{T}(X, Y)=\frac{(1-q) X-q Y}{1-2 q}, \\
& S(X, Y)=\frac{(1-q) Y-q X}{1-2 q} .
\end{aligned}
$$

Condition (7) is given by

$$
\begin{aligned}
D & =\left\{(X, Y): X^{2}+4 q Y>-4 q^{2}, \quad X<0, \quad Y<-q\right\} \\
& =\left\{(X, Y): X<0,-\frac{X^{2}}{4 q}-q<Y<-q\right\} .
\end{aligned}
$$

The domain $D$ is illustrated in Fig. 2.

We apply Lemma 1 to find the joint distribution of $X$ and $Y$. We compute the Jacobian of the transform $(X, Y) \rightarrow(\hat{T}, S)$, which is given by

$$
\begin{aligned}
J & =\left(\begin{array}{ll}
\frac{\partial \hat{T}(X, Y)}{\partial X} & \frac{\partial S(X, Y)}{\partial Y} \\
\frac{\partial \hat{T}(X, Y)}{\partial X} & \frac{\partial S(X, Y)}{\partial Y}
\end{array}\right) \\
& =\left(\begin{array}{ll}
\frac{1-q}{1-2 q} & \frac{-q}{1-2 q} \\
\frac{-q}{1-2 q} & \frac{1-q}{1-2 q}
\end{array}\right) \\
& =\frac{1}{1-2 q} .
\end{aligned}
$$


Hence if $(\hat{T}, S)$ has a probability density $f(t, s)$ then $(X, Y)$ has a probability density

$$
g(x, y)=|J| f(\hat{T}(x, y), S(x, y)) .
$$

We now apply this approach to SH and PD games.

\subsection{The Stag Hunt (SH)}

Proposition 1 The probability that $S H$ games have 3 equilibria is given by

$$
p_{3}^{\mathrm{SH}}=\frac{1}{(1-2 q)} \operatorname{Area}\left(D \cap D_{1}\right),
$$

where $D$ is the subset of $\mathbb{R}^{2}$ determined by

$$
D=\left\{(x, y):-1<x<0,-\frac{x^{2}}{4 q}-q<y<-q\right\},
$$

and $D_{1}$ is the quadrilateral $A B O C$ with vertices

$$
\begin{aligned}
& A=(-1,-1), \quad B=(-(1-q),-q), \\
& C=(-q,-(1-q)) \quad O=(0,0) .
\end{aligned}
$$

The domain $D_{1}$ and the intersection $D \cap D_{1}$ is illustrated in Fig. 3.

Proof In the SH game: $1>T>0>S>-1, T \sim$ $U(0,1), S \sim U(-1,0)$. Let $\hat{T}:=T-1 \sim U(-1,0)$. The joint distribution of $(\hat{T}, S)$ is

$$
f(t, s)= \begin{cases}1 & (t, s) \in(-1,0) \times(-1,0), \\ 0 & \text { otherwise }\end{cases}
$$

According to (10), the joint probability distribution of $(X, Y)$ is

$$
\begin{aligned}
g(x, y) & =|J| f(\hat{T}(x, y), S(x, y)) \\
& =\frac{1}{(1-2 q)} 1_{(x, y) \in D_{1}},
\end{aligned}
$$

where

$$
\begin{aligned}
D_{1}= & (\hat{T}(x, y), S(x, y) \in(-1,0) \times(-1,0) \\
= & \left((x, y) \in(-1,0)^{2}:(1-q) x-q y,(1-q) y-q x\right) \\
& \in(-(1-2 q), 0) \times(-(1-2 q), 0) \\
= & \left\{(x, y) \in(-1,0)^{2}:-(1-2 q)\right. \\
< & (1-q) x-q y<0, \\
& -(1-2 q)<(1-q) y-q x<0\} \\
= & \left\{(x, y) \in(-1,0)^{2}: \frac{(1-q) x}{q}\right. \\
< & y<\frac{(1-q) x+(1-2 q)}{q}
\end{aligned}
$$

$$
\left.\frac{q x-(1-2 q)}{1-q}<y<\frac{q x}{1-q}\right\} .
$$

We can characterise $D_{1}$ further by explicitly ordering the lower and upper bounds in the above formula. We have

$$
\begin{aligned}
& \frac{(1-q) x}{q}-\frac{q x}{1-q}=\frac{(1-2 q) x}{q(1-q)}<0 \\
& \frac{(1-q) x}{q}-\frac{q x-(1-2 q)}{1-q}=\frac{(1-2 q)(x+q)}{q(1-q)}, \\
& \frac{(1-q) x+(1-2 q)}{q}-\frac{q x}{1-q}=\frac{(1-2 q)(x+1-q)}{q(1-q)} .
\end{aligned}
$$

It follows that:

(i) for $-1<x<-(1-q)<-q$ :

$$
\begin{aligned}
\frac{(1-q) x}{q} & <\frac{q x-(1-2 q)}{1-q} \\
& <\frac{(1-q) x+(1-2 q)}{q} \\
& <\frac{q x}{1-q}
\end{aligned}
$$

(ii) For $-(1-q)<x<-q$

$$
\begin{aligned}
\frac{(1-q) x}{q} & <\frac{q x-(1-2 q)}{1-q} \\
& <\frac{q x}{1-q} \\
& <\frac{(1-q) x+(1-2 q)}{q} .
\end{aligned}
$$

(iii) for $-q<x<0$

$$
\begin{aligned}
\frac{q x-(1-2 q)}{1-q} & <\frac{(1-q) x}{q} \\
& <\frac{q x}{1-q} \\
& <\frac{(1-q) x+(1-2 q)}{q} .
\end{aligned}
$$

Hence

$$
\begin{aligned}
D_{1}= & \left\{-1<x<-(1-q), \frac{q x-(1-2 q)}{1-q}<y\right. \\
< & \left.\frac{(1-q) x+(1-2 q)}{q}\right\} \\
& \cup\{-(1-q)<x<-q, \\
& \left.\frac{q x-(1-2 q)}{1-q}<y<\frac{q x}{1-q}\right\}
\end{aligned}
$$



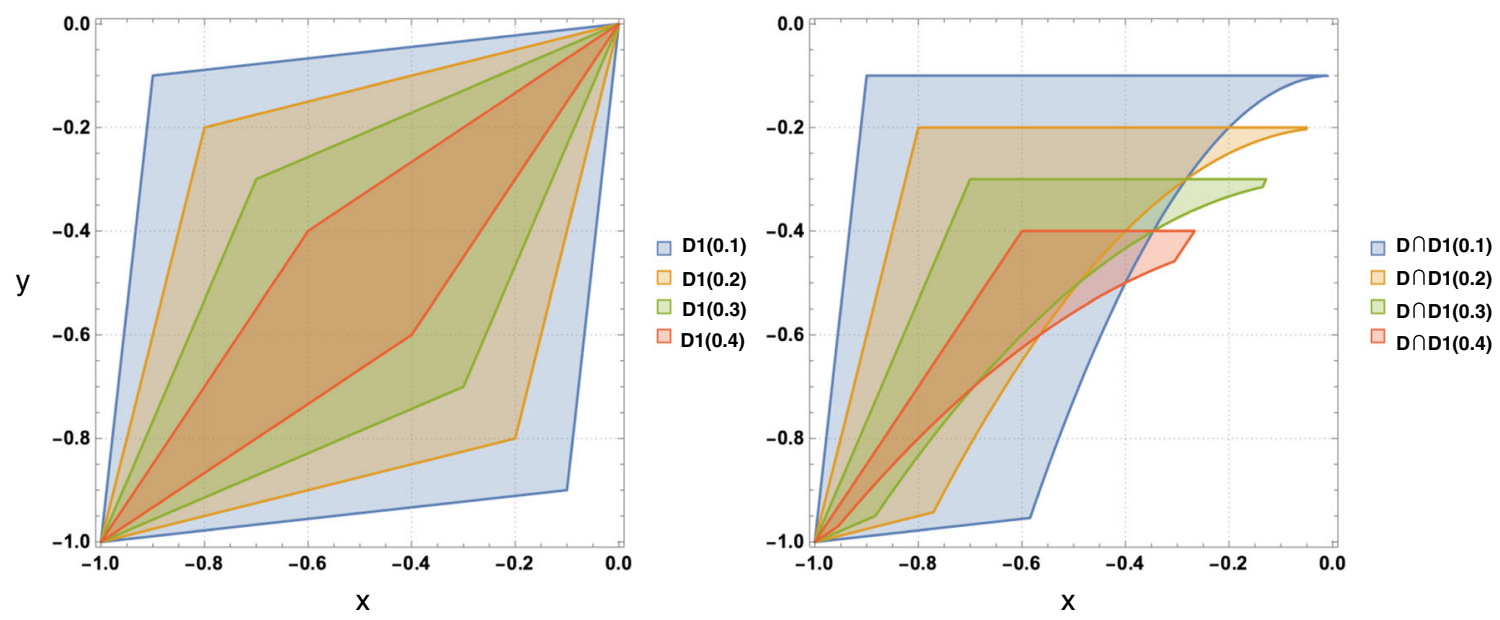

Fig. 3 SH game: regions $D_{1}$ and $D \cap D_{1}$ are shown for different values of $q$, namely, $q=0.1,0.2,0.3$ and 0.4 , for illustration (see Proposition 1)

$$
\begin{aligned}
& \cup\{-q<x<-0, \\
& \left.\frac{(1-q) x}{q}<y<\frac{q x}{1-q}\right\} .
\end{aligned}
$$

Thus, $D_{1}$ is the quadrilateral $A B O C$ with vertices

$$
\begin{aligned}
& A=(-1,-1), \quad B=(-(1-q),-q), \\
& C=(-q,-(1-q)) \quad O=(0,0) .
\end{aligned}
$$

Theorem 3 The probability that $S H$ games have 3 equilibria is given by other hand, the parabola $y=-\frac{x^{2}}{4 q}-q$ meets the line $y=\frac{q x-(1-2 q)}{1-q}$ at two points $G_{1}=\left(x_{1},-\frac{x_{1}^{2}}{4 q}-q\right)$ and $G_{2}=\left(x_{2},-\frac{x_{2}^{2}}{4 q}-q\right)$ with

$$
\begin{aligned}
& x_{1}=2 q\left(-\frac{q}{1-q}-\frac{2 q-1}{(q-1) \sqrt{q}}\right), \\
& x_{2}=2 q\left(\frac{2 q-1}{(q-1) \sqrt{q}}-\frac{q}{1-q}\right) .
\end{aligned}
$$

By comparing $x_{1}$ and $x_{2}$ with $-q$ we have $G_{1}$ is always in the edge $A C$, while $G_{2}$ is outside $A C$ if $0<q \leq 4 / 9$ and is inside $A C$ if $4 / 9<q<0.5$.

In conclusion

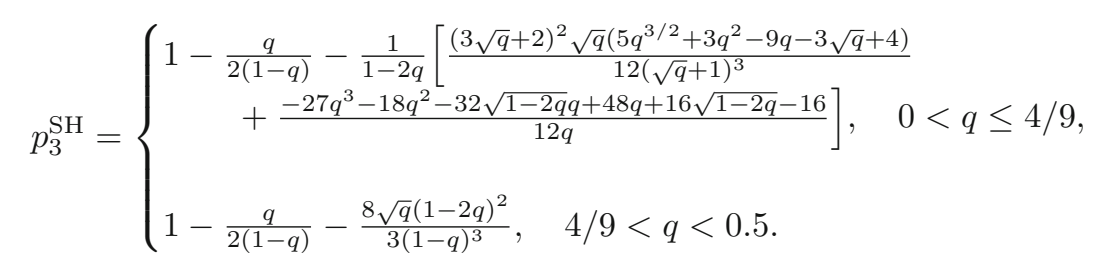

Proof According to Proposition 1, in order to compute $p_{3}^{\mathrm{SH}}$ we need to compute the area of $D \cap D_{1}$. To this end we need to understand the intersections of the parabola $y=-\frac{x^{2}}{4 q}-q$ with the lines $y=\frac{(1-q) x}{q}$ and $y=\frac{q x-(1-2 q)}{1-q}$. The parabola $y=-\frac{x^{2}}{4 q}-q$ always intersects with the line $y=\frac{(1-q) x}{q}$ inside the domain $(-1,0)^{2}$ at the point

$$
F=(2(q+\sqrt{1-2 q}-1), 2(1-q)(q+\sqrt{1-2 q}-1) / q)
$$

By comparing $2(q+\sqrt{1-2 q}-1)$ with $-q$, it follows that the point $F$ is inside the edge $O C$ if $0 \leq q \leq 4 / 9$ and is outside $O C$ whenever $4 / 9<q<0 . \overline{5}$. On the
1. for $0<q \leq 4 / 9$ : the intersection $D \cap D_{1}$ is the domain formed by vertices $A, B, E, F$, and $G_{1}$ where $E=\left(-\frac{q^{2}}{1-q},-q\right)$ (which is the intersection of $y=-q$ with $\left.y=\frac{(1-q) x}{q}\right)$ and

- $A$ and $B$ are connected by the line $y=$ $\frac{(1-q) x+(1-2 q)}{q}$,

- $B$ and $E$ are connected by the line $y=-q$,

- $E$ and $F$ are connected by the line $y=\frac{(1-q) x}{q}$,

- $F$ and $G_{1}$ are connected by the parabola $y=$ $-\frac{x^{2}}{4 q}-q$,

- $G_{1}$ and $A$ are connected by the line $y=$ $\frac{q x-(1-2 q)}{1-q}$. 
2. for $4 / 9<q<0.5$ : the intersection $D \cap D_{1}$ is the domain formed by the vertices $A, B, E, C, G_{1}, G_{2}$ where

- $A$ and $B$ are connected by the line $y=$ $\frac{(1-q) x+(1-2 q)}{q}$,

- $B$ and $E$ are connected by the line $y=-q$,

- $E$ and $C$ are connected by the line $y=\frac{(1-q) x}{q}$,

- $C$ and $G_{1}$ are connected by the line $y=$ $\frac{q x-(1-2 q)}{1-q}$,

- $G_{1}$ and $G_{2}$ are connected by the parabola $y=$ $-\frac{x^{2}}{4 q}-q$,

- $G_{2}$ and $A$ are connected by the line $y=$ $\frac{q x-(1-2 q)}{1-q}$.

See Fig. 4 for illustrations of the two cases above, with $q=0.3$ and $q=0.45$ respectively.

We are now ready to compute the probability that the $\mathrm{SH}$ game has three equilibria.

For $0<q \leq 4 / 9$ : The probability that the $\mathrm{SH}$ game has three equilibria is

$$
\begin{aligned}
p_{3}^{\mathrm{SH}}= & \frac{1}{(1-2 q)} \operatorname{Area}\left(D \cap D_{1}\right) \\
= & \frac{1}{(1-2 q)}\left[\operatorname{Area}\left(D_{1}\right)\right. \\
& \left.-\operatorname{Area}(B O E)-\operatorname{Area}\left(C F G_{1}\right)\right]
\end{aligned}
$$

We proceed by computing the areas in the expression above. Area of $D_{1}$ is

$$
\begin{aligned}
\operatorname{Area} & \left(D_{1}\right) \\
= & \int_{-1}^{-(1-q)}\left[\frac{(1-q) x+(1-2 q)}{q}\right. \\
- & \left.\frac{q x-(1-2 q)}{1-q}\right] \mathrm{d} x \\
& +\int_{-(1-q)}^{-q}\left[\frac{q x}{1-q}-\frac{q x-(1-2 q)}{1-q}\right] \mathrm{d} x \\
& +\int_{-q}^{0}\left[\frac{q x}{1-q}-\frac{(1-q) x}{q}\right] \mathrm{d} x \\
= & \frac{q(1-2 q)}{2(1-q)}+\frac{(1-2 q)^{2}}{1-q} \\
& +\frac{q(1-2 q)}{2(1-q)} \\
= & 1-2 q .
\end{aligned}
$$

Area of $B O E$ is

$$
\begin{aligned}
\operatorname{Area}(B O E) & =\operatorname{Area}(B O H)-\operatorname{Area}(H O E) \\
& =\frac{1}{2} q\left[(1-q)-\frac{q^{2}}{1-q}\right] \\
& =\frac{q(1-2 q)}{2(1-q)}
\end{aligned}
$$

where $H=(0,-q)$. Area of $C F G$ is

$$
\begin{aligned}
\operatorname{Area}( & \left.C F G_{1}\right) \\
= & \int_{a}^{-q}\left[\frac{q x-(1-2 q)}{1-q}+\frac{x^{2}}{4 q}+q\right] \mathrm{d} x \\
& +\int_{-q}^{2(q+\sqrt{1-2 q}-1)}\left[\frac{(1-q) x}{q}+\frac{x^{2}}{4 q}+q\right] \mathrm{d} x \\
= & \frac{(3 \sqrt{q}+2)^{2} \sqrt{q}\left(5 q^{3 / 2}+3 q^{2}-9 q-3 \sqrt{q}+4\right)}{12(\sqrt{q}+1)^{3}} \\
& +\frac{-27 q^{3}-18 q^{2}-32 \sqrt{1-2 q}+48 q+16 \sqrt{1-2 q}-16}{12 q} .
\end{aligned}
$$

Substituting (13), (14), and (15) back to (12) we obtain, for $0<q \leq 4 / 9$

$$
\begin{aligned}
p_{3}^{\mathrm{SH}}= & 1-\frac{q}{2(1-q)} \\
& -\frac{1}{1-2 q}\left[\frac{(3 \sqrt{q}+2)^{2} \sqrt{q}\left(5 q^{3 / 2}+3 q^{2}-9 q-3 \sqrt{q}+4\right)}{12(\sqrt{q}+1)^{3}}\right. \\
& \left.+\frac{-27 q^{3}-18 q^{2}-32 \sqrt{1-2 q} q+48 q+16 \sqrt{1-2 q}-16}{12 q}\right]
\end{aligned}
$$

Now we consider the remaining case $4 / 9<q<0.5$. In this case

$$
\begin{aligned}
p_{3}^{\mathrm{SH}}= & \frac{1}{(1-2 q)} \operatorname{Area}\left(D \cap D_{1}\right) \\
= & \frac{1}{(1-2 q)}\left[\operatorname{Area}\left(D_{1}\right)\right. \\
& \left.-\operatorname{Area}(B O E)-\operatorname{Area}\left(\diamond G_{1} G_{2}\right)\right],
\end{aligned}
$$

where $\diamond G_{1} G_{2}$ is the domain with vertices $G_{1}$ and $G_{2}$ formed by the parabola $y=-\frac{x^{2}}{4 q}-q$ and the line $y=$ $\frac{q x-(1-2 q)}{1-q}$. Thus

$$
\begin{aligned}
\operatorname{Area}\left(\diamond G_{1} G_{2}\right) & =\int_{x_{1}}^{x_{2}}\left[-\frac{x^{2}}{4 q}-q-\frac{q x-(1-2 q)}{1-q}\right] \mathrm{d} x \\
& =\frac{8 \sqrt{q}(1-2 q)^{3}}{3(1-q)^{3}}
\end{aligned}
$$

Substituting (13), (14) and (17) back to (16) we obtain, for $4 / 9<q<0.5$,

$$
p_{3}^{\mathrm{SH}}=1-\frac{q}{2(1-q)}-\frac{8 \sqrt{q}(1-2 q)^{2}}{3(1-q)^{3}} .
$$

This finishes the proof of this theorem

\subsection{Prisoner's Dilemma (PD)}

Proposition 2 The probability that PD games have 3 equilibria is given by

$$
p_{3}^{\mathrm{PD}}=\frac{1}{(1-2 q)} \operatorname{Area}\left(D \cap D_{2}\right),
$$



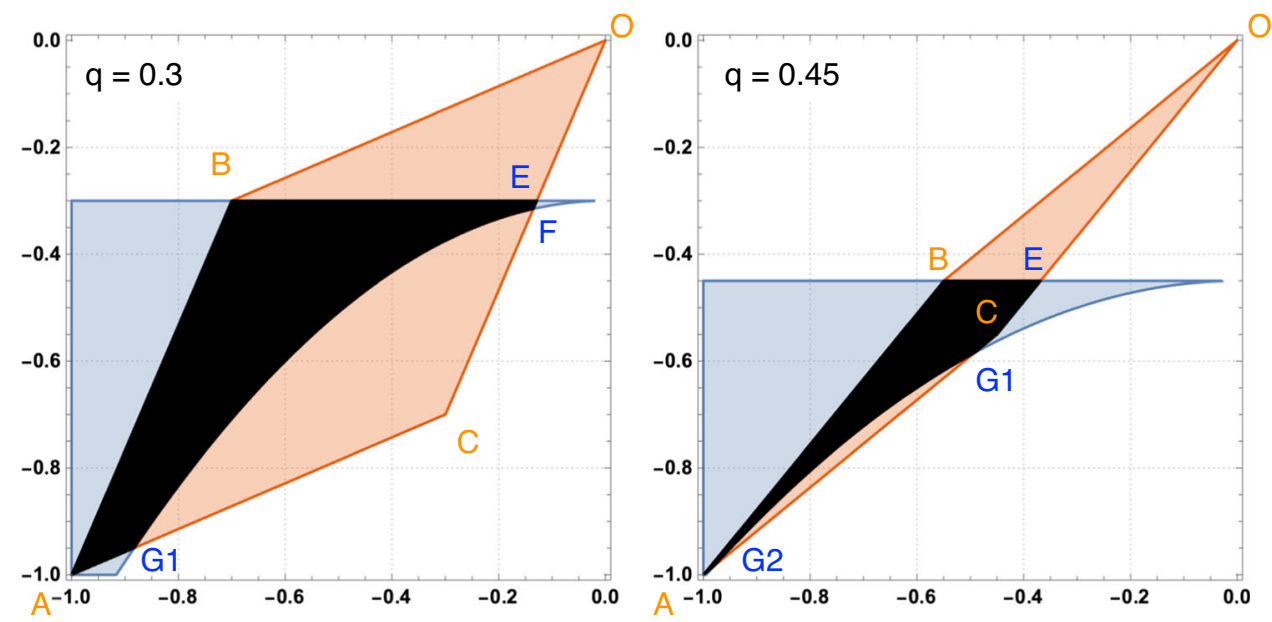

Fig. 4 Details of $D \cap D_{1}$ for $q=0.3$ and $q=0.45$, for illustration of the proof and calculation in Theorem 3

where $D$ is defined above (as in the case of the $\mathrm{SH}$ games) and $D_{2}$ is the triangle $M N O$ with vertices

$M=(-q,-(1-q)), \quad N=\left(0,-\frac{1-2 q}{1-q}\right), \quad O=(0,0)$.

See Fig. 5 for illustration of $D_{2}$ and its intersection with $D$ for several values of $q$.

Proof Recall that in PD games we have $T \sim U(1,2)$, $S \sim(-1,0)$. Thus $\hat{T}=T-1 \sim(0,1)$. The joint distribution of $(\hat{T}, S)$ is

$$
\begin{aligned}
f(t, s) & =1_{(x, y) \in(0,1) \times(-1,0)} \\
& = \begin{cases}1 & (t, s) \in(0,1) \times(-1,0), \\
0 & \text { otherwise }\end{cases}
\end{aligned}
$$

According to (10), the joint probability distribution of $(X, Y)$ is

$$
\begin{aligned}
g(x, y) & =|J| f(\hat{T}(x, y), S(x, y)) \\
& =\frac{1}{(1-2 q)} 1_{(x, y) \in D_{2}},
\end{aligned}
$$

where

$$
\begin{aligned}
D_{2}= & (\hat{T}(x, y), S(x, y) \in(0,1) \times(-1,0) \\
= & \left\{(x, y) \in(-1,0)^{2}:(1-q) x-q y,(1-q) y-q x\right) \\
& \in(0,1-2 q) \times(-(1-2 q), 0)\} \\
= & \left\{(x, y) \in(-1,0)^{2}: 0<(1-q) x-q y<1-2 q,\right. \\
& -(1-2 q)<(1-q) y-q x<0\} \\
= & \left\{(x, y) \in(-1,0)^{2}: \frac{(1-q) x-(1-2 q)}{q}\right. \\
& <y<\frac{(1-q) x}{q},
\end{aligned}
$$

$$
\left.\frac{q x-(1-2 q)}{1-q}<y<\frac{q x}{1-q}\right\} \text {. }
$$

We now characterise $D_{2}$ further. We have

(1) for $-1<x<-q$ then

$$
\begin{aligned}
\frac{(1-q) x-(1-2 q)}{q} & <\frac{(1-q) x}{q} \\
& <\frac{q x-(1-2 q)}{1-q} \\
& <\frac{q x}{1-q}
\end{aligned}
$$

(2) for $-q<x<0$ then

$$
\begin{aligned}
\frac{(1-q) x-(1-2 q)}{q} & <\frac{q x-(1-2 q)}{1-q} \\
& <\frac{(1-q) x}{q} \\
& <\frac{q x}{1-q}
\end{aligned}
$$

It follows that

$$
\begin{aligned}
D_{2}= & \left\{(x, y):-q<x<0, \frac{q x-(1-2 q)}{1-q}\right. \\
& \left.<y<\frac{(1-q) x}{q}\right\} .
\end{aligned}
$$

Thus $D_{2}$ is the triangle $M N O$ with vertices

$$
\begin{aligned}
& M=(-q,-(1-q)), \\
& N=\left(0,-\frac{1-2 q}{1-q}\right), \quad O=(0,0) .
\end{aligned}
$$



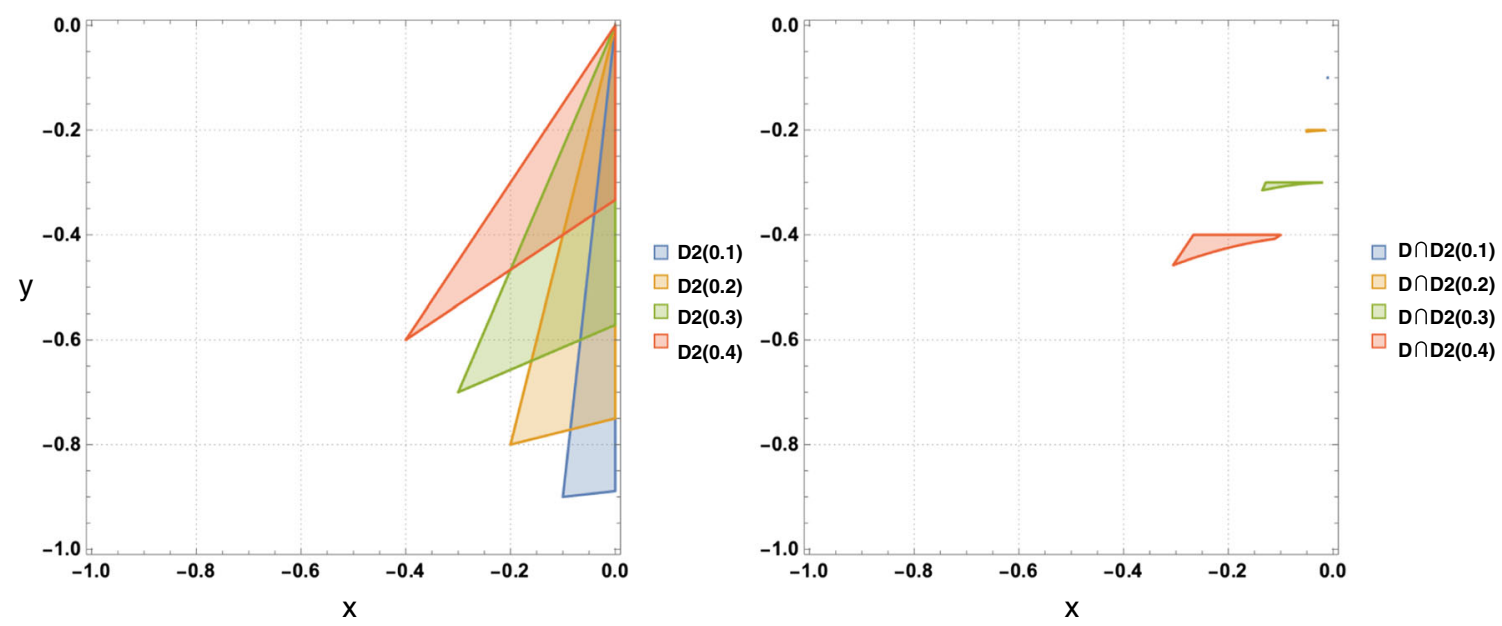

Fig. 5 PD game: regions $D_{2}$ and $D \cap D_{2}$ are shown for different values of $q$, namely, $q=0.1,0.2,0.3$ and 0.4 , for illustration of Proposition 2

The probability that the $\mathrm{SH}$ game has three equilibria is thus

$$
\begin{aligned}
p_{3}^{\mathrm{SH}} & =\int_{D} g(x, y) \mathrm{d} x \mathrm{~d} y \\
& =\frac{1}{(1-2 q)} \int_{D} 1_{(x, y) \in D_{2}} \mathrm{~d} x \mathrm{~d} y \\
& =\frac{1}{(1-2 q)} \operatorname{Area}\left(D \cap D_{2}\right)
\end{aligned}
$$

This finishes the proof of this proposition.

The following elementary lemma provides an upper bound for $p_{3}^{\mathrm{PD}}$, particularly implying that it tends to 0 as $q$ goes to 0 .

\section{Lemma 2}

$$
p_{3}^{\mathrm{PD}} \leq \frac{q}{2(1-q)}
$$

As a consequence, $p_{3}^{\mathrm{PD}}$ is always smaller or equal to 0.5 and tends to 0 as $q$ tends to 0 .

$$
p_{3}^{\mathrm{PD}}=\left\{\begin{array}{l}
\frac{1}{1-2 q}\left[-\frac{2\left(q^{3}+3 q^{2}+(4 \sqrt{1-2 q}-6) q-2 \sqrt{1-2 q}+2\right)}{3 q}-\frac{1}{2} \frac{q^{3}}{(1-q)}\right], \quad 0 \leq q \leq \frac{3-\sqrt{5}}{2}, \\
\frac{-16(\sqrt{1-2 q}-1) q^{3 / 2}+2 q^{5 / 2}+15 q^{3}+(8 \sqrt{1-2 q}-25) q^{2}+q-8 \sqrt{1-2 q}+2 \sqrt{q}(8 \sqrt{1-2 q}-5)+5}{6(\sqrt{q}-1)^{3}\left(q^{3 / 2}+q\right)}, \quad \frac{3-\sqrt{5}}{2}<q \leq 4 / 9 \\
\frac{1}{2} \frac{(1-2 q)^{2}}{q(1-q)}, \quad 4 / 9<q<0.5 .
\end{array}\right.
$$

Proof Area of $D$

$$
\begin{aligned}
\operatorname{Area}(D) & =\int_{-1}^{0}\left[-q-\left(-\frac{x^{2}}{4 q}-q\right)\right] \mathrm{d} x \\
& =\frac{1}{12 q} .
\end{aligned}
$$

Area of $D_{2}$

$$
\begin{aligned}
\operatorname{Area}\left(D_{2}\right) & =\int_{-q}^{0}\left[\frac{(1-q) x}{q}-\frac{q x-(1-2 q)}{1-q}\right] \mathrm{d} x \\
& =\frac{q(1-2 q)}{2(1-q)} .
\end{aligned}
$$

Hence

$$
\begin{aligned}
p_{3}^{\mathrm{PD}} & \leq \frac{1}{1-2 q} \min \left\{\operatorname{Area}(D), \operatorname{Area}\left(D_{2}\right)\right\} \\
& =\frac{1}{1-2 q} \min \left\{\frac{1}{12 q}, \frac{q(1-2 q)}{2(1-q)}\right\} \\
& =\frac{q}{2(1-q)} .
\end{aligned}
$$

Theorem 4 The probability that PD games has three equilibria is given by 

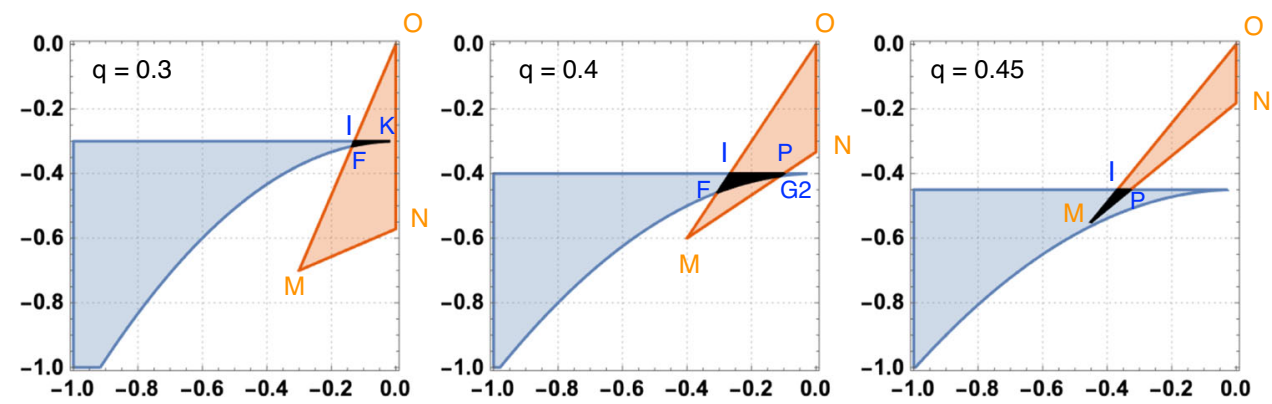

Fig. 6 Details of $D \cap D_{2}$ for $q=0.3,0.4,0.45$, for illustration of the proof and calculation in Theorem 4

Proof According to Proposition 2, in order to compute $p_{3}^{\mathrm{PD}}$ we need to compute the area of $D \cap D_{2}$. As in proof of Theorem 3, the parabola $y=-\frac{x^{2}}{4 q}-q$ always intersects with the line $y=\frac{(1-q) x}{q}$ inside the domain $(-1,0)^{2}$ at the point

$F=(2(q+\sqrt{1-2 q}-1), 2(1-q)(q+\sqrt{1-2 q}-1) / q)$.

The point $F$ is inside the edge $D C$ if $0 \leq q \leq 4 / 9$ and is outside $D C$ whenever $4 / 9<q<0.5$.

On the other hand, the parabola $y=-\frac{x^{2}}{4 q}-q$ meets the line $y=\frac{q x-(1-2 q)}{1-q}$ at two points $G_{1}=\left(x_{1},-\frac{x_{1}^{2}}{4 q}-q\right)$ and $G_{2}=\left(x_{2},-\frac{x_{2}^{2}}{4 q}-q\right)$ with

$$
\begin{aligned}
& x_{1}=2 q\left(-\frac{q}{1-q}-\frac{1-2 q}{(1-q) \sqrt{q}}\right), \\
& x_{2}=2 q\left(-\frac{q}{1-q}+\frac{1-2 q}{(1-q) \sqrt{q}}\right) .
\end{aligned}
$$

$G_{1}$ is always outside edge $A C . G_{2}$ is inside it if $\frac{3-\sqrt{5}}{2}<$ $q \leq 4 / 9$ and outside it otherwise. Therefore, we have three cases (see Fig. 6 for illustration).

(1) For $0 \leq q \leq \frac{3-\sqrt{5}}{2}$ : the intersection $D \cap D_{2}$ is formed by $I, F, K$ where $I=\left(-q^{2} /(1-q),-q\right)$ (which is the intersection of $y=-q$ with $y=$ $\left.\frac{(1-q) x}{q}\right), K=(0 .-q)$ and

- $I$ and $F$ are connected by the line $y=\frac{(1-q) x}{q}$,

$-F$ and $K$ are connected by the parabola $y=$ $-\frac{x^{2}}{4 q}-q$,

- $K$ and $I$ are connected by the line $y=-q$.

In this case

$$
\begin{aligned}
p_{3}^{\mathrm{PD}} & =\frac{1}{1-2 q} \operatorname{Area}\left(D \cap D_{2}\right) \\
& =\frac{1}{1-2 q}(\operatorname{Area}(O F K)-\operatorname{Area}(O I K))
\end{aligned}
$$

$$
\begin{aligned}
= & \frac{1}{1-2 q}\left(\int _ { 2 ( q + \sqrt { 1 - 2 q } - 1 ) } ^ { 0 } \left[\frac{(1-q) x}{q}\right.\right. \\
& \left.\left.+\frac{x^{2}}{4 q}+q\right] \mathrm{~d} x-\frac{1}{2} \frac{q^{3}}{(1-q)}\right) \\
= & \frac{1}{1-2 q}\left[-\frac{2\left(q^{3}+3 q^{2}+(4 \sqrt{1-2 q}-6) q-2 \sqrt{1-2 q}+2\right)}{3 q}\right. \\
& \left.-\frac{1}{2} \frac{q^{3}}{(1-q)}\right] .
\end{aligned}
$$

(2) For $\frac{3-\sqrt{5}}{2}<q \leq 4 / 9$ : the intersection $D \cap D_{2}$ is formed by $F, I, P=\left(\left(q^{3}-3 q+1\right) / q,-q\right)$ (which is the intersection of $y=-q$ with $\left.y=\frac{q x-(1-2 q)}{1-q}\right)$ and $G_{2}$, where

- $F$ and $I$ are connected by the line $y=\frac{(1-q) x}{q}$,

- $I$ and $P$ are connected by the line $y=-q$,

- $P$ and $G_{2}$ are connected by the line $y=$ $\frac{q x-(1-2 q)}{1-q}$,

- $G_{2}$ and $F$ are connected by the parabola $y=$ $-\frac{x^{2}}{4 q}-q$.

In this case

$$
\begin{aligned}
p_{3}^{\mathrm{PD}}= & \frac{1}{1-2 q} \operatorname{Area}\left(D \cap D_{2}\right) \\
= & \frac{1}{1-2 q}\left(\int _ { 2 ( q + \sqrt { 1 - 2 q } - 1 ) } ^ { - \frac { q ^ { 2 } } { 1 - q } } \left[\frac{(1-q) x}{q}\right.\right. \\
& \left.+\frac{x^{2}}{4 q}+q\right] \mathrm{d} x+\int_{-\frac{q^{2}}{1-q}}^{x_{2}} \frac{x^{2}}{4 q} \mathrm{~d} x \\
& \left.+\int_{x_{2}}^{\left(q^{2}-3 q+1\right) / q}\left[-q-\frac{q x-(1-2 q)}{1-q}\right] \mathrm{d} x\right) \\
= & \frac{1}{6(\sqrt{q}-1)^{3}\left(q^{3 / 2}+q\right)}\left(-16(\sqrt{1-2 q}-1) q^{3 / 2}\right. \\
& +2 q^{5 / 2}+15 q^{3}+(8 \sqrt{1-2 q}-25) q^{2} \\
& +q-8 \sqrt{1-2 q}+2 \sqrt{q}(8 \sqrt{1-2 q}-5)+5) .
\end{aligned}
$$


(3) For $4 / 9<q<0.5$ : the intersection $D \cap D_{2}$ is the triangle $M I P$ where $M=(-q,-(1-q))$

- $M$ and $I$ are connected by the line $y=\frac{(1-q) x}{q}$,

- $I$ and $P$ are connected by the line $y=-q$,

- $P$ and $M$ are connected by the line $y=$ $\frac{q x-(1-2 q)}{1-q}$.

In this case

$$
\begin{aligned}
p_{3}^{\mathrm{PD}}= & \frac{1}{1-2 q} \operatorname{Area}\left(D \cap D_{2}\right) \\
= & \frac{1}{1-2 q} \operatorname{Area}(M I P) \\
= & \frac{1}{2(1-2 q)}\left(\frac{q^{2}-3 q+1}{q}\right. \\
& \left.+\frac{q^{2}}{1-q}\right)(1-2 q) \\
= & \frac{1}{2} \frac{(1-2 q)^{2}}{q(1-q)} .
\end{aligned}
$$

results have clearly shown the influence of the mutation on the number of equilibria in SH-games and PDgames. The probability distributions in these games are much more complicated than in SD-games and H-games and significantly depend on the mutation strength. For a summary of our results, see again Box 1 and Fig. 1. Our analysis has made use of suitable changes of variables, which expressed the probability densities in terms of area of certain domains. For future work, we plan to generalise our method to other approaches to studying random social dilemmas such as finite population dynamics and payoff disturbances $[2,3,16,36]$, as well as to multi-player $[21,27,33]$ and more complex social dilemma games such as the climate change and technological race interactions $[14,35]$.

Acknowledgements TAH is supported by Leverhulme Research Fellowship (RF-2020-603/9).

In conclusion

$$
p_{3}^{\mathrm{PD}}=\left\{\begin{array}{l}
\frac{1}{1-2 q}\left[-\frac{2\left(q^{3}+3 q^{2}+(4 \sqrt{1-2 q}-6) q-2 \sqrt{1-2 q}+2\right)}{3 q}-\frac{1}{2} \frac{q^{3}}{(1-q)}\right], \quad 0 \leq q \leq \frac{3-\sqrt{5}}{2}, \\
\frac{-16(\sqrt{1-2 q}-1) q^{3 / 2}+2 q^{5 / 2}+15 q^{3}+(8 \sqrt{1-2 q}-25) q^{2}+q-8 \sqrt{1-2 q}+2 \sqrt{q}(8 \sqrt{1-2 q}-5)+5}{6(\sqrt{q}-1)^{3}\left(q^{3 / 2}+q\right)}, \quad \frac{3-\sqrt{5}}{2}<q \leq 4 / 9 \\
\frac{1}{2} \frac{(1-2 q)^{2}}{q(1-q)}, \quad 4 / 9<q<0.5
\end{array}\right.
$$

This completes the proof of this theorem.

\section{Summary and outlook}

It has been shown that in many social and, more generally, biological settings mutation is non-negligible $[30,37,40]$. How mutation affects the complexity and bio-diversity of the evolutionary systems is a fundamental question in evolutionary dynamics $[24,32]$. In this paper, we have addressed this question for random social dilemmas by computing explicitly the probability distributions of the number of equilibria in term of the mutation probability. Our analysis based on random games is highly relevant and practical, because it is often the case that one might know the nature of a game at hand (e.g., a coordination or cooperation dilemma), but it is very difficult and/or costly to measure the exact values of the game's payoff matrix. Our

\section{Author contributions}

The authors contributed equally to this paper.

Data Availability Statement This manuscript has no associated data or the data will not be deposited. [Authors' comment: This is a theoretical research, no data has been used.]

Open Access This article is licensed under a Creative Commons Attribution 4.0 International License, which permits use, sharing, adaptation, distribution and reproduction in any medium or format, as long as you give appropriate credit to the original author(s) and the source, provide a link to the Creative Commons licence, and indicate if changes were made. The images or other third party material in this article are included in the article's Creative Commons licence, unless indicated otherwise in a credit line to the material. If material is not included in the article's Creative Commons licence and your intended use is not permitted by statutory regulation or exceeds the permitted use, you will need to obtain permission directly from the copyright holder. To view a copy of this licence, visit http://creativecomm ons.org/licenses/by/4.0/. 


\section{References}

1. C. Adami, J. Schossau, A. Hintze, Evolutionary game theory using agent-based methods. Phys. Life Rev. 19, $1-26$ (2016)

2. M. A Amaral, M.A. Javarone, Heterogeneity in evolutionary games: an analysis of the risk perception. Proc. R. Soc. A, 476, 20200116 (2020)

3. M.A. Amaral, M.A. Javarone, Strategy equilibrium in dilemma games with off-diagonal payoff perturbations. Phys. Rev. E 101, 062309 (2020)

4. M. Broom, Evolutionary games with variable payoffs. C. R. Biol. 328, 403-412 (2005)

5. M. Broom, C. Cannings, G.T. Vickers, On the number of local maxima of a constrained quadratic form. Proc. R. Soc. Lond. A 443, 573-584 (1993)

6. M.H. Duong, T.A. Han, On the expected number of equilibria in a multi-player multi-strategy evolutionary game. Dyn. Games Appl. 6, 324-346 (2016)

7. M.H. Duong, T.A. Han, Analysis of the expected density of internal equilibria in random evolutionary multiplayer multi-strategy games. J. Math. Biol. 73(6), 17271760 (2016)

8. M.H. Duong, T.A. Han, On equilibrium properties of the replicator-mutator equation in deterministic and random games. Dyn. Games Appl. 10(3), 641-663 (2020)

9. M.H. Duong, H.M. Tran, T.A. Han, On the distribution of the number of internal equilibria in random evolutionary games. J. Math. Biol. 78(1), 331-371 (2019)

10. R. Durrett, The Essentials of Probability (Duxbury Press, Pacific Grove, 1994)

11. C.S. Gokhale, A. Traulsen, Evolutionary games in the multiverse. Proc. Natl. Acad. Sci. USA 107(12), 55005504 (2010)

12. K.P. Hadeler, Stable polymorphisms in a selection model with mutation. SIAM J. Appl. Math. 41(1), 1-7 (1981)

13. T.A. Han, L.M. Pereira, F.C. Santos, T. Lenaerts, Good agreements make good friends. Sci. Rep. 3(1), 1-7 (2013)

14. T.A. Han, L.M. Pereira, F.C. Santos, T. Lenaerts, To regulate or not: a social dynamics analysis of an idealised AI race. J. Artif. Intell. Res. 69, 881-921 (2020)

15. T.A. Han, A. Traulsen, C.S. Gokhale, On equilibrium properties of evolutionary multi-player games with random payoff matrices. Theor. Popul. Biol. 81(4), 264-272 (2012)

16. W. Huang, A. Traulsen, Fixation probabilities of random mutants under frequency dependent selection. J. Theor. Biol. 263, 262-268 (2010)

17. L.A. Imhof, D. Fudenberg, M.A. Nowak, Evolutionary cycles of cooperation and defection. Proc. Natl. Acad. Sci. 102(31), 10797-10800 (2005)

18. N.L. Komarova, Replicator-mutator equation, universality property and population dynamics of learning. J. Theor. Biol. 230(2), 227-239 (2004)

19. N.L. Komarova, S.A. Levin, Eavesdropping and language dynamics. J. Theor. Biol. 264(1), 104-118 (2010)

20. N.L. Komarova, P. Niyogi, M.A. Nowak, The evolutionary dynamics of grammar acquisition. J. Theor. Biol. 209(1), 43-59 (2001)
21. Q. Luo, L. Liu, X. Chen, Evolutionary dynamics of cooperation in the n-person stag hunt game. Physica D 424, 132943 (2021)

22. R.M. May, Stability and complexity in model ecosystems, vol. 6 (Princeton University Press, Princeton, 2001)

23. J.M. McNamara, Towards a richer evolutionary game theory. J. R. Soc. Interface 10(88), 20130544 (2013)

24. M.A. Nowak, Evolutionary Dynamics (Harvard University Press, Cambridge, 2006)

25. M.A. Nowak, N.L. Komarova, P. Niyogi, Evolution of universal grammar. Science 291(5501), 114-118 (2001)

26. R. Olfati-Saber, Evolutionary dynamics of behavior in social networks. In: 2007 46th IEEE Conference on Decision and Control, pp. 4051-4056 (2007)

27. J.M. Pacheco, F.C. Santos, M.O. Souza, B. Skyrms, Evolutionary dynamics of collective action in n-person stag hunt dilemmas. Proc. R. Soc. Lond. B Biol. Sci. 276(1655), 315-321 (2009)

28. D. Pais, C. Caicedo-Nún?z, N. Leonard, Hopf bifurcations and limit cycles in evolutionary network dynamics. SIAM J. Appl. Dyn. Syst. 11(4), 1754-1784 (2012)

29. M. Perc, J.J. Jordan, D.G. Rand, Z. Wang, S. Boccaletti, A. Szolnoki, Statistical physics of human cooperation. Phys. Rep. 687, 1-51 (2017)

30. D.G. Rand, C.E. Tarnita, H. Ohtsuki, M.A. Nowak, Evolution of fairness in the one-shot anonymous ultimatum game. Proc. Natl. Acad. Sci. 110(7), 2581-2586 (2013)

31. F.C. Santos, J.M. Pacheco, T. Lenaerts, Evolutionary dynamics of social dilemmas in structured heterogeneous populations. Proc. Natl. Acad. Sci. USA 103, 3490-3494 (2006)

32. K. Sigmund, The Calculus of Selfishness (Princeton Univ. Press, Princeton, 2010)

33. M.O. Souza, J.M. Pacheco, F.C. Santos, Evolution of cooperation under n-person snowdrift games. J. Theor. Biol. 260(4), 581-588 (2009)

34. P.F. Stadler, P. Schuster, Mutation in autocatalytic reaction networks. J. Math. Biol. 30(6), 597-632 (1992)

35. W. Sun, L. Liu, X. Chen, A. Szolnoki, V.V. Vasconcelos, Combination of institutional incentives for cooperative governance of risky commons. iScience 24(8), 102844 (2021)

36. A. Szolnoki, M. Perc, Seasonal payoff variations and the evolution of cooperation in social dilemmas. Sci. Rep. 9(1), 1-9 (2019)

37. A. Traulsen, C. Hauert, H. De Silva, M.A. Nowak, K. Sigmund, Exploration dynamics in evolutionary games. Proc. Natl. Acad. Sci. USA 106, 709-712 (2009)

38. X. Wang, M. Duh, M. Perc, Robust cooperation against mutations via costly expulsion. EPL (Europhys. Lett.) 132(3), 38001 (2020)

39. Z. Wang, S. Kokubo, M. Jusup, J. Tanimoto, Universal scaling for the dilemma strength in evolutionary games. Phys. Life Rev. 14, 1-30 (2015)

40. I. Zisis, S. Di Guida, T.A. Han, G. Kirchsteiger, T. Lenaerts, Generosity motivated by acceptanceevolutionary analysis of an anticipation game. Sci. Rep. 5(1), 1-11 (2015) 